Pacific

Journal of

Mathematics

\title{
SIGNATURES OF LEFSCHETZ FIBRATIONS
}

\author{
BURAK OzBAGCI
}




\title{
SIGNATURES OF LEFSCHETZ FIBRATIONS
}

\author{
BuraK OzBAGCI
}

\begin{abstract}
Let $M$ be a smooth 4-manifold which admits a Lefschetz fibration over $D^{2}$ or $S^{2}$. We develop an algorithm to compute the signature of $M$ using the global monodromy of this fibration. As a corollary we prove that there is no hyperelliptic Lefschetz fibration over $S^{2}$ with only reducible singular fibers.
\end{abstract}

\section{Introduction.}

The signature of a smooth 4-manifold which admits a hyperelliptic Lefschetz fibration of genus $g$ over a closed surface can be computed using the local signature formula given by Matsumoto ([M1], [M2]) for $g=1,2$ and more recently extended by Endo $[\mathbf{E}]$ for $g \geq 3$.

In this paper we present an algorithm to compute the signature of a smooth 4-manifold which admits an arbitrary (not necessarily hyperelliptic) Lefschetz fibration of any genus over $D^{2}$ or $S^{2}$. A Lefschetz fibration on a smooth 4-manifold $M$ gives rise to a handlebody description of $M$, which is determined by a sequence of vanishing cycles. We use this handlebody description $[\mathbf{K}]$ and Wall's nonadditivity formula for signatures $[\mathbf{W}]$ to compute the signature of $M$. Hence we calculate a 'signature contribution' corresponding to each singular fiber of the given fibration on $M$.

As a corollary we prove that "there is no hyperelliptic Lefschetz fibration over $S^{2}$ with only reducible singular fibers." After we proved and announced this result the general case (not assuming the hyperellipticity) was proved independently by $\mathrm{Li}[\mathbf{L}]$, Smith $[\mathbf{S m}]$ and Stipsicz $[\mathbf{S t 3}]$ all using a result of this paper (cf. Corollary 7).

Recent results in symplectic topology show that Lefschetz fibrations provide a topological characterization of symplectic 4-manifolds: Donaldson [D] has shown that, after perhaps blowing up, a closed symplectic 4-manifold admits a Lefschetz fibration over $S^{2}$, and conversely Gompf [GS] has shown that most Lefschetz fibrations are symplectic - the exceptions all have fibergenus one and are blow-ups of torus fibrations with no critical points. Hence by computing the signatures of Lefschetz fibrations we hope to attack some of the problems in the geography of symplectic 4-manifolds ([St1], [St2], $[\mathrm{GS}])$. 
We also prove that the signature of a smooth 4-manifold which admits a hyperelliptic Lefschetz fibration of genus $g \leq 3$ over $S^{2}$ is nonpositive. It is conjectured that this is true for all genus $g$ Lefschetz fibrations over $S^{2}$.

We want to point out that despite the fact that the vanishing cycles are defined up to isotopy, our technique shows that the signature of a 4-manifold which admits a Lefschetz fibration depends only on the algebraic data given by the homology classes of the vanishing cycles.

In $[\mathbf{S m}]$, Smith gave an elegant signature formula using the geometry of Lefschetz fibrations. Even though his formula is in a closed form, it seems impossible to actually compute the signature using his formula.

Acknowledgement. The author would like to thank John Etnyre, Terry Fuller, Ludmil Katzarkov, Ron Stern and Richard Wentworth for helpful conversations. In particular, the author would like to thank Terry Fuller for stimulating his interest in Lefschetz fibrations. The author would also like to thank Yukio Matsumoto for informing him about the preprint $[\mathbf{E}]$ by Hisaaki Endo. The author would like to thank Andras Stipsicz for commenting on earlier versions of this paper. Finally, the author wishes to express his gratitute to Ron Stern for encouragement and many useful discussions.

\section{Preliminaries.}

1.1. Mapping class groups. Let $\Sigma_{g}$ be a closed oriented surface of genus $g$. Let $\operatorname{Diff}^{+}\left(\Sigma_{g}\right)$ be the group of all orientation preserving self diffeomorphisms of $\Sigma_{g}$. Let Diff ${ }_{0}^{+}\left(\Sigma_{g}\right)$ be the subgroup of $\operatorname{Diff}^{+}\left(\Sigma_{g}\right)$ consisting of all self diffeomorphisms isotopic to the identity. Then we define the mapping class group of genus $g$ as

$$
\mathcal{M}_{g}=\operatorname{Diff}^{+}\left(\Sigma_{g}\right) / \operatorname{Diff}_{0}^{+}\left(\Sigma_{g}\right) .
$$

The hyperelliptic mapping class group $\mathcal{H}_{g}$ of genus $g$ is defined as the subgroup of $\mathcal{M}_{g}$ which consists of all isotopy classes commuting with the isotopy class of the hyperelliptic involution $\iota: \Sigma_{g} \longrightarrow \Sigma_{g}$.

It is known that the hyperelliptic mapping class group $\mathcal{H}_{g}$ agrees with the mapping class group $\mathcal{M}_{g}$ for $g=1,2$ (cf. $[\mathbf{B H}]$ ).

A positive (or right-handed) Dehn twist $D(\alpha): \Sigma_{g} \rightarrow \Sigma_{g}$ about a simple closed curve $\alpha$ is a diffeomorphism obtained by cutting $\Sigma_{g}$ along $\alpha$, twisting $360^{\circ}$ to the right and regluing. Note that the positive Dehn twist $D(\alpha)$ is determined up to isotopy by $\alpha$ and is independent of the orientation on $\alpha$.

It is well-known that the mapping class group $\mathcal{M}_{g}$ is generated by Dehn twists.

We will use the functional notation for the products in $\mathcal{M}_{g}$, e.g., $D(\beta) D(\alpha)$ will denote the composition where we apply $D(\alpha)$ first and then $D(\beta)$. 


\subsection{Smooth Lefschetz fibrations.}

Let $M$ be a compact, oriented smooth 4-manifold, and let $B$ be a compact, oriented 2-manifold. A proper smooth map $f: M \rightarrow B$ is a smooth Lefschetz fibration if there exist points $b_{1}, \ldots, b_{m} \in \operatorname{interior}(B)$ such that

(1) $\left\{b_{1}, \ldots, b_{m}\right\}$ are the critical values of $f$, with $p_{i} \in f^{-1}\left(b_{i}\right)$ a unique critical point of $f$, for each $i$, and

(2) about each $b_{i}$ and $p_{i}$, there are local complex coordinate charts agreeing with the orientations of $M$ and $B$ such that locally $f$ can be expressed as $f\left(z_{1}, z_{2}\right)=z_{1}^{2}+z_{2}^{2}$.

Remark. An achiral Lefschetz fibration is a fibration which satisfies (1) and (2) above without requiring the coincidence of the canonical orientation determined by $\left(z_{1}, z_{2}\right)$ and the orientation of $M$.

It is a consequence of the definition of a smooth Lefschetz fibration that

$$
\left.f\right|_{f^{-1}\left(B-\left\{b_{1}, \ldots, b_{m}\right\}\right)}: f^{-1}\left(B-\left\{b_{1}, \ldots, b_{m}\right\}\right) \rightarrow B-\left\{b_{1}, \ldots, b_{m}\right\}
$$

is a smooth fiber bundle over $B-\left\{b_{1}, \ldots, b_{m}\right\}$ with fiber diffeomorphic to a 2-manifold $\Sigma_{g}$, and so we refer to $f$ (and sometimes also the manifold $M$ ) as a genus $g$ Lefschetz fibration (or a Lefschetz fibration of genus $g$ ). Two genus $g$ Lefschetz fibrations $f: M \rightarrow B$ and $f^{\prime}: M^{\prime} \rightarrow B^{\prime}$ are equivalent if there are diffeomorphisms $\Phi: M \rightarrow M^{\prime}$ and $\phi: B \rightarrow B^{\prime}$ such that $f^{\prime} \Phi=\phi f$.

We always assume that our Lefschetz fibrations are relatively minimal, namely that no fiber contains an embedded 2-sphere of self-intersection number -1 . We also assume that there is at least one singular fiber in each fibration.

If $f: M \rightarrow D^{2}$ is a smooth genus $g$ Lefschetz fibration, then we can use this fibration to produce a handlebody description of $M$. We select a regular value $b_{0} \in \operatorname{interior}\left(D^{2}\right)$ of $f$, an identification $f^{-1}\left(b_{0}\right) \cong \Sigma_{g}$, and a collection of arcs $s_{i}$ in interior $\left(D^{2}\right)$ with each $s_{i}$ connecting $b_{0}$ to $b_{i}$, and otherwise disjoint from the other arcs. We also assume that the critical values are indexed so that the $\operatorname{arcs} s_{1}, \ldots, s_{m}$ appear in order as we travel counterclockwise in a small circle about $b_{0}$. Let $V_{0}, \ldots, V_{m}$ denote a collection of small disjoint open disks with $b_{i} \in V_{i}$ for each $i$.

To build our description of $M$, we observe first that $f^{-1}\left(V_{0}\right) \cong \Sigma_{g} \times D^{2}$, with $\partial\left(f^{-1}\left(V_{0}\right)\right) \cong \Sigma_{g} \times S^{1}$. Let $\nu\left(s_{i}\right)$ be a regular neighborhood of the arc $s_{i}$. Enlarging $V_{0}$ to include the critical value $b_{1}$, it can be shown that $f^{-1}\left(V_{0} \cup\right.$ $\left.\nu\left(s_{1}\right) \cup V_{1}\right)$ is diffeomorphic to $\Sigma_{g} \times D^{2}$ with a 2-handle $h_{1}$ attached along a circle $\gamma_{1}$ contained in a fiber $\Sigma_{g} \times p t \subset \Sigma_{g} \times S^{1}$. Moreover, Condition (2) in the definition of a Lefschetz fibration requires that $h_{1}$ is attached with a framing -1 relative to the natural framing on $\gamma_{1}$ inherited from the product structure of $\partial\left(f^{-1}\left(V_{0}\right)\right) \cdot \gamma_{1}$ is called a vanishing cycle. In addition, $\partial\left(\left(\Sigma_{g} \times D^{2}\right) \cup h_{1}\right)$ is diffeomorphic to a $\Sigma_{g}$-bundle over $S^{1}$ whose monodromy is given by $D\left(\gamma_{1}\right)$, 


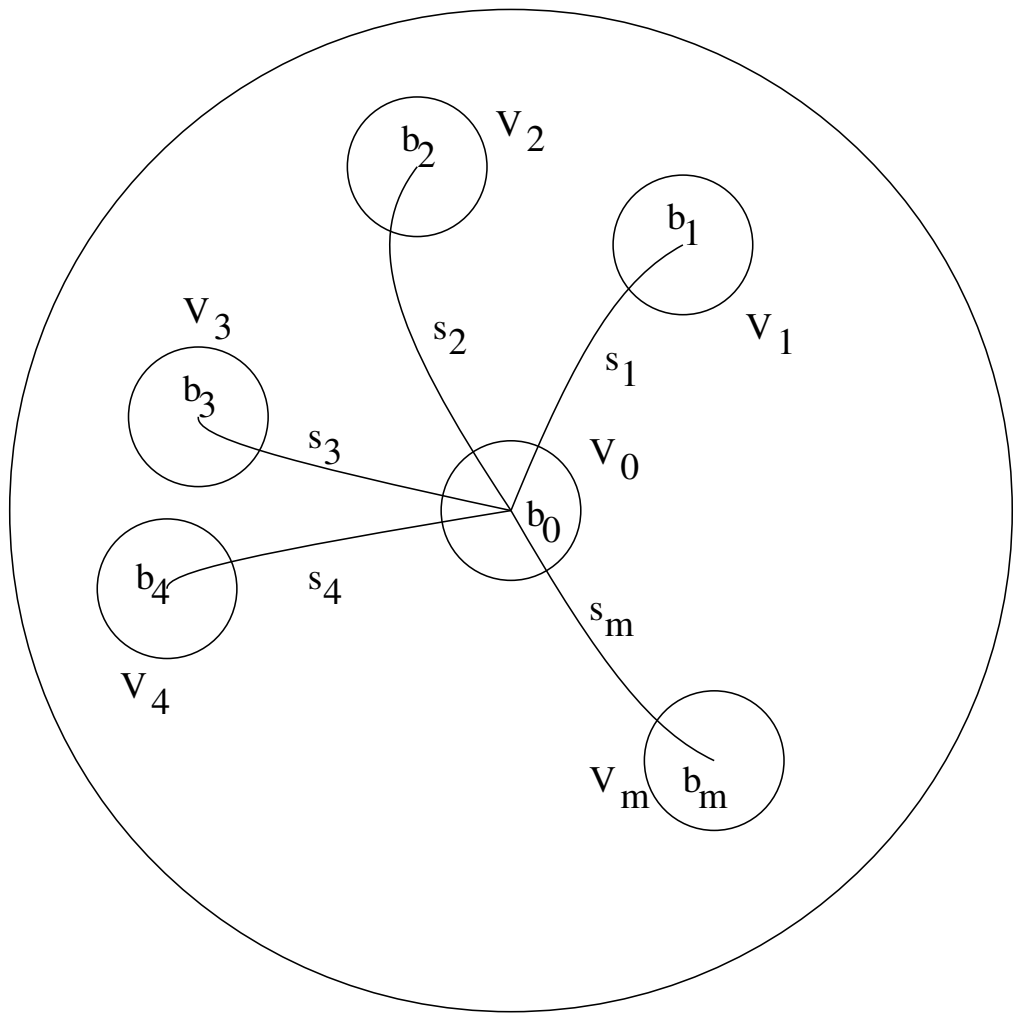

Figure 1. Fibration over the disk.

a positive Dehn twist about $\gamma_{1}$. Continuing counterclockwise about $b_{0}$, we add the remaining critical values to our description, yielding that

$$
M_{0} \cong f^{-1}\left(V_{0} \cup\left(\bigcup_{i=1}^{m} \nu\left(s_{i}\right)\right) \cup\left(\bigcup_{i=1}^{m} V_{i}\right)\right)
$$

is diffeomorphic to $\left(\Sigma_{g} \times D^{2}\right) \cup\left(\bigcup_{i=1}^{m} h_{i}\right)$, where each $h_{i}$ is a 2-handle attached along a vanishing cycle $\gamma_{i}$ in a $\Sigma_{g}$-fiber in $\Sigma_{g} \times S^{1}$ with relative framing -1 . This handle attaching procedure will be explained in more detail in Section 2. Furthermore,

$$
\partial M_{0} \cong \partial\left(\left(\Sigma_{g} \times D^{2}\right) \cup\left(\bigcup_{i=1}^{m} h_{i}\right)\right)
$$

is a $\Sigma_{g}$-bundle over $S^{1}$ with monodromy given by the composition $D\left(\gamma_{m}\right)$ $\cdots D\left(\gamma_{1}\right)$. We will refer to the cyclically ordered collection $\left(D\left(\gamma_{1}\right), \ldots\right.$, $D\left(\gamma_{m}\right)$ ) (or the product $D\left(\gamma_{m}\right) \cdots D\left(\gamma_{1}\right)$ ) as the global monodromy of this fibration. 
A Lefschetz fibration $f: M \rightarrow D^{2}$ does not completely determine the ordered collection $\left(D\left(\gamma_{1}\right), \ldots, D\left(\gamma_{m}\right)\right)$. Aside from the cyclic permutations and being able to conjugate all elements by a fixed arbitrary element of $\Gamma_{g}$, different choices of $\left\{s_{i}\right\}$ will give different monodromies. Given two choices of $\left\{s_{i}\right\}$, it is possible to get between them by a sequence of moves and their inverses. These moves which are called elementary transformations, can be thought of as the Lefschetz analog of handle slides in Morse theory. Each move interchanges the corresponding vanishing cycles, and also acts on one of the two cycles by the monodromy of the other. Equivalently, the pair of Dehn twists $\left(D\left(\gamma_{i}\right), D\left(\gamma_{i+1}\right)\right)$ is replaced by $\left(D\left(\gamma_{i+1}\right), D\left(\gamma_{i+1}\right) D\left(\gamma_{i}\right) D\left(\gamma_{i+1}\right)^{-1}\right)$. Thus, two relatively minimal Lefschetz fibrations over $D^{2}$ will be equivalent if and only if it is possible to get between the corresponding ordered collections of monodromies by a sequence of elementary transformations (and their inverses), together with an inner automorphism of $\mathcal{M}_{g}$ (cf. [GS]).

We can extend this description to Lefschetz fibrations over $S^{2}$ as follows:

Assume that $f: M \rightarrow S^{2}$ is a smooth genus $g$ Lefschetz fibration. Let $M_{0}=M-\nu\left(f^{-1}(b)\right)$, where $\nu\left(f^{-1}(b)\right) \cong \Sigma_{g} \times D^{2}$ denotes a regular neighborhood of a nonsingular fiber $f^{-1}(b)$. Then $\left.f\right|_{M_{0}}: M_{0} \rightarrow D^{2}$ is a smooth Lefschetz fibration. If $\left(D\left(\gamma_{1}\right), \ldots, D\left(\gamma_{m}\right)\right)$ is the global monodromy of the fibration $\left.f\right|_{M_{0}}: M_{0} \rightarrow D^{2}$, then $D\left(\gamma_{m}\right) \cdots D\left(\gamma_{1}\right)$ is isotopic to the identity since also $\partial M_{0} \cong \Sigma_{g} \times S^{1}$. Finally, to extend our description of $M_{0}$ to $M$, we reattach $\Sigma_{g} \times D^{2}$ to $\left(\Sigma_{g} \times D^{2}\right) \cup\left(\bigcup_{i=1}^{m} h_{i}\right)$ via a $\Sigma_{g}$-fiber preserving map of the boundary. This extension is unique up to equivalence for $g \geq 2[\mathbf{K}]$.

Definition. Let $f: M \rightarrow S^{2}$ be a smooth genus $g$ Lefschetz fibration with global monodromy $\left(D\left(\gamma_{1}\right), \ldots, D\left(\gamma_{m}\right)\right)$. We will call $f: M \rightarrow S^{2}$ a hyperelliptic Lefschetz fibration of genus $g$ iff there exists $h \in \mathcal{M}_{g}$ such that $h D\left(\gamma_{i}\right) h^{-1} \in \mathcal{H}_{g}$ for all $\mathrm{i}, 1 \leq i \leq m$.

Remark. All Lefschetz fibrations of genus one and genus two are hyperelliptic since $\mathcal{H}_{g}=\mathcal{M}_{g}$ for $g=1,2$.

\subsection{Wall's non-additivity formula.}

If two compact oriented 4-manifolds are glued by an orientation reversing diffeomorphism of their boundaries, then the signature of their union is the sum of their signatures. This is known as the Novikov additivity. But it is often desirable to consider the more general case of gluing: Along a common submanifold, which may itself have boundary, of the boundaries of the original manifolds. However, the Novikov additivity does not hold in this general case. Wall $[\mathbf{W}]$ derives a formula for the deviation from additivity in the general case, which is known as the Wall's nonadditivity formula.

We will give a specific case of his formula:

Let $X_{-}, X_{0}, X_{+}$be 3-manifolds and $Y_{-}$and $Y_{+}$be 4-manifolds such that $\partial X_{-}=\partial X_{0}=\partial X_{+}=Z$,

$\partial Y_{-}=X_{-} \cup X_{0}$ 
$\partial Y_{+}=X_{0} \cup X_{+}$

write $Y=Y_{-} \cup Y_{+}$and $X=X_{-} \cup X_{0} \cup X_{+}$(Figure 2).

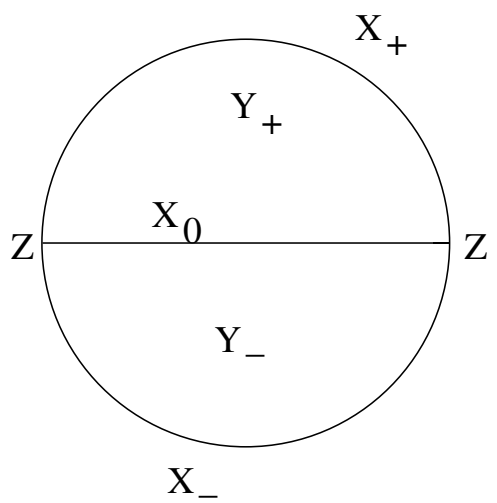

Figure 2.

Suppose that $Y$ is oriented inducing orientations of $Y_{-}$and $Y_{+}$. Orient the rest so that

$\partial_{*}\left[Y_{-}\right]=\left[X_{0}\right]-\left[X_{-}\right]$,

$\partial_{*}\left[Y_{+}\right]=\left[X_{+}\right]-\left[X_{0}\right]$,

$\partial_{*}\left[X_{-}\right]=\partial_{*}\left[X_{0}\right]=\partial_{*}\left[X_{+}\right]=[Z]$.

Write $V=H_{1}(Z ; \mathbb{R})$; let $A, B$ and $C$ be the kernels of the maps on first homology induced by the inclusions of $Z$ in $X_{-}, X_{0}$ and $X_{+}$respectively. Then $\operatorname{dim} A=\operatorname{dim} B=\operatorname{dim} C=\frac{(\operatorname{dim} V)}{2}$.

Let $\Phi$ denote the oriented intersection pairing in $Z$. Note that $A, B$ and $C$ are maximal isotropic subspaces for the intersection pairing $\Phi$. Let $W=$ $\frac{C \cap(A+B)}{(C \cap A)+(C \cap B)}$. Wall $[\mathbf{W}]$ defines a symmetric bilinear map $\Psi: W \times W \rightarrow \mathbb{R}$ as follows: The map $\Psi^{\prime}: C \cap(A+B) \times C \cap(A+B) \rightarrow \mathbb{R}$ defined by $\Psi^{\prime}\left(c, c^{\prime}\right)=\Phi\left(c, a^{\prime}\right)$ where $a^{\prime}+b^{\prime}+c^{\prime}=0$ for some $a^{\prime} \in A$ and $b^{\prime} \in B$ induces a well-defined bilinear map $\Psi$ on $W$.

The signature of the symmetric bilinear map $\Psi$ will be denoted by $\sigma(V$; $C, A, B)$.

We also denote the signature of a 4-manifold $\mathrm{M}$ as $\sigma(M)$ in the rest of this paper.

We are now ready to state Wall's formula:

Theorem $1([\mathbf{W}]) \cdot \sigma(Y)=\sigma\left(Y_{-}\right)+\sigma\left(Y_{+}\right)-\sigma(V ; C, A, B)$.

\subsection{Local signature formula.}

The following theorem was proven by Matsumoto for $g=1,2$ using the fact that the cohomology class of Meyer's signature cocyle has finite order in the cohomology group $H^{2}\left(\mathcal{M}_{g}, \mathbb{Z}\right)$. Recently, Endo proved the $g \geq 3$ case by 
observing the finiteness of the order of the cohomology class of the signature cocycle restricted to the hyperelliptic mapping class group $\mathcal{H}_{g}$.

Theorem 2 ([M1], [M2], [E]). Let $M$ be a 4-manifold which admits a hyperelliptic Lefschetz fibration of genus $g$ over $S^{2}$. Let $n$ and $s=\sum_{h=1}^{\left[\frac{g}{2}\right]} s_{h}$ be the numbers of nonseparating and separating vanishing cycles in the global monodromy of this fibration, respectively. Then

$$
\sigma(M)=-\frac{g+1}{2 g+1} n+\sum_{h=1}^{\left[\frac{g}{2}\right]}\left(\frac{4 h(g-h)}{2 g+1}-1\right) s_{h} .
$$

Remarks. (1) Here $s_{h}$ denotes the number of separating vanishing cycles which separate the genus $g$ surface into two surfaces one of which has genus $h$.

(2) This formula was reproven for the case $g=2$ in $[\mathbf{S m}]$.

\section{Main theorems.}

In this section we explain our main idea and establish the main theorems to develop an algorithm to compute the signature of a 4-manifold which admits a Lefschetz fibration over $D^{2}$ or $S^{2}$ using the global monodromy of this fibration.

Definition. Let $X$ be a 4-manifold with boundary $\partial X \cong \Sigma_{g} \times I /(x, 1) \sim$ $(\phi(x), 0)$, where $\phi$ is a self-diffeomorphism of $\Sigma_{g}$. Let $X^{\prime}$ denote the resulting 4-manifold after attaching a 2-handle to $X$ along a simple closed curve $\gamma$ on $\Sigma_{g} \times\{p t\}$ with framing -1 (relative to the product framing). Then $\sigma(\phi, \gamma)$ is defined as $\sigma\left(X^{\prime}\right)-\sigma(X)$.

Theorem 3. Let $M$ be a 4-manifold which admits a genus $g$ Lefschetz fibration over $D^{2}$ or $S^{2}$. Let $\left(D\left(\gamma_{1}\right), \ldots, D\left(\gamma_{t}\right)\right)$ be the global monodromy of this fibration. Let $D\left(\gamma_{0}\right)$ denote the identity map. Then

$$
\sigma(M)=\Sigma_{i=1}^{t} \sigma\left(D\left(\gamma_{i-1}\right) \cdots D\left(\gamma_{0}\right), \gamma_{i}\right),
$$

where $\sigma\left(D\left(\gamma_{i-1}\right) \cdots D\left(\gamma_{1}\right), \gamma_{i}\right) \in\{-1,0,+1\}$ for all $i, 1 \leq i \leq t$.

Proof. It suffices to prove the result for Lefschetz fibrations over $D^{2}$. (By Novikov additivity it extends to Lefschetz fibrations over $S^{2}$.) We use the handlebody description of $M$ and Wall's formula as follows:

We start with a copy of $M_{0}=\Sigma_{g} \times D^{2}$. We attach a 2-handle to $M_{0}$ along $\gamma_{1}$ with framing -1 . Let $M_{1}$ denote the resulting manifold. Then $\partial M_{1}$ will have monodromy $D\left(\gamma_{1}\right)$, a positive Dehn twist about $\gamma_{1}$. Now we attach another 2-handle to $M_{1}$ along $\gamma_{2}$. Let $M_{2}$ denote the resulting manifold. Proceeding in this manner we get the manifolds $M_{1}, M_{2}, \ldots, M_{t}$. 
We are going to apply Wall's formula at each step of this contruction to compute the signature of $M$. In order to apply Wall's formula we set up the following notation:

Take $\phi, X, \gamma$ and $X^{\prime}$ as in the definition above.

Let $\nu(\gamma)$ denote a regular neighborhood of $\gamma$ in $\partial X$, and let $i_{*}$ be the induced map on the homology by the inclusion of appropriate spaces.

Now we define $Y_{+}, Y_{-}, X_{+}, X_{0}, X_{-}, Z$ in Wall's formula as follows:

$$
\begin{aligned}
& Y_{-}=D^{2} \times D^{2}, \quad Y_{+}=X \\
& \partial Y_{-}=\partial\left(D^{2} \times D^{2}\right)=S^{1} \times D^{2} \cup D^{2} \times S^{1}, \quad \partial Y_{+}=\Sigma_{g} \times I /(x, 1) \sim(\phi(x), 0), \\
& X_{0}=S^{1} \times D^{2} \cong \nu(\gamma), \quad X_{-}=D^{2} \times S^{1}, \quad X_{+}=\partial X-\stackrel{\circ}{\nu}(\gamma), \\
& Z=S^{1} \times S^{1} \cong \partial \nu(\gamma) \cong \partial(\partial X-\stackrel{\circ}{\nu}(\gamma)) .
\end{aligned}
$$

Hence,

$$
\begin{aligned}
& A=\operatorname{Ker}\left(i_{*}: H_{1}\left(S^{1} \times S^{1} ; \mathbb{R}\right) \rightarrow H_{1}\left(D^{2} \times S^{1} ; \mathbb{R}\right)\right), \\
& B=\operatorname{Ker}\left(i_{*}: H_{1}\left(S^{1} \times S^{1} ; \mathbb{R}\right) \rightarrow H_{1}\left(S^{1} \times D^{2} ; \mathbb{R}\right)\right), \\
& C=\operatorname{Ker}\left(i_{*}: H_{1}(\partial \nu(\gamma) ; \mathbb{R}) \rightarrow H_{1}(\partial X-\stackrel{\circ}{(\gamma)} ; \mathbb{R})\right) .
\end{aligned}
$$

Let $l$ be the longitude $S^{1} \times\{p t\}$ and $m$ be the meridian $\{p t\} \times \partial D^{2}$ of $X_{0}=S^{1} \times D^{2}$. Then $A=\langle[l]\rangle$ and $B=\langle[m]\rangle$. We also know that $C$ is a 1dimensional subspace of

$$
H_{1}\left(S^{1} \times S^{1} ; \mathbb{R}\right)=\langle[l],[m]\rangle \cong \mathbb{R}^{2} .
$$

Let $\Phi$ be the intersection form on $Z=S^{1} \times S^{1}$ and $W=\frac{C \cap(A+B)}{(C \cap A)+(C \cap B)}=$ $\frac{C}{(C \cap A)+(C \cap B)}$. Hence $W=\{0\}$ if $C=A$ or $C=B$ and $W=C$ otherwise. Now assume that $C \neq A$ and $C \neq B$. Then $C=\langle c\rangle=\langle p[l]+q[m]\rangle$ for some $p, q \in \mathbb{R}$ and $\Psi(c, c)=\Phi\left(c, a^{\prime}\right)$ where $c+a^{\prime}+b^{\prime}=0$ for some $a^{\prime} \in A$ and $b^{\prime} \in B$. ( $\Psi$ is the bilinear form in Wall's formula). Let $a^{\prime}=-p[l]$ and $b^{\prime}=-q[m]$. Then we have,

$$
\Psi(c, c)=\Phi(c,-p[l])=\Phi(p[l]+q[m],-p[l])=-p q \Phi([m],[l])=p q .
$$

Therefore signature of $\Psi$ is given by the sign of $p q$.

Hence by Wall's formula

$$
\begin{aligned}
\sigma\left(X^{\prime}\right) & =\sigma(X)+\sigma\left(D^{2} \times D^{2}\right)-\sigma\left(\mathbb{R}^{2} ; C, A, B\right) \\
& =\sigma(X)-\operatorname{signature}(\Psi)=\sigma(X)-\operatorname{sign}(p q) .
\end{aligned}
$$

This proves the theorem by setting $X=M_{i}$ for $i=1,2, \ldots, t-1$.

So the idea to compute the signature of a genus $g$ Lefschetz fibration is very simple. For each 2 -handle that we attach to $\Sigma_{g} \times D^{2}$ along a vanishing cycle, there is a corresponding signature contribution $\in\{-1,0,+1\}$. Once we attach all the 2-handles, the sum of the signature contributions will be signature of the 4-manifold. The difficulty is to compute the signature contributions using the vanishing cycles (or more precisely using only the 
homology classes of the vanishing cycles). The following technical theorems will be helpful in computations.

Theorem 4. In addition to the notation above, let $\left\{a_{1}, b_{1}, a_{2}, b_{2}, \ldots, a_{g}, b_{g}\right\}$ be the standard basis for $H_{1}\left(\Sigma_{g} ; \mathbb{R}\right)$. (We will use the letters $a_{i}$ and $b_{i}$ also to denote the curves which represent the homology classes $a_{i}$ and $b_{i}$, respectively, for $1 \leq i \leq g$.) Then:

(1) If $\gamma$ is a nonseparating curve, then there exists a longitude $l^{\prime}$ and a meridian $m^{\prime}$ of $\partial(\partial X-\stackrel{\circ}{\nu}(\gamma))$ such that

$$
\begin{gathered}
i_{*}\left[l^{\prime}\right]=[\gamma] \in H_{1}(\partial X-\stackrel{\circ}{\nu}(\gamma) ; \mathbb{R}) \\
i_{*}\left[m^{\prime}\right]=\frac{e-\phi_{*}(e)}{e \cdot[\gamma]} \in H_{1}(\partial X-\stackrel{\circ}{\nu}(\gamma) ; \mathbb{R})
\end{gathered}
$$

for all $e \in\left\{a_{1}, b_{1}, a_{2}, b_{2}, \ldots, a_{g}, b_{g}\right\}$, where $e \cdot[\gamma] \neq 0$.

(2) If $\gamma$ is a separating curve, then $\sigma\left(X^{\prime}\right)=\sigma(X)-1$, i.e., $\sigma(\phi, \gamma)=-1$.

Proof. We recall that $\partial X$ is a mapping torus, i.e., $\partial X \cong \Sigma_{g} \times I /(x, 1) \sim$ $(\phi(x), 0)$ and $\gamma$ is a curve on a fiber $\Sigma_{g} \times\{p t\}$. We note that a regular neighborhood of $\gamma$ in $\Sigma_{g}$ is given by $\gamma \times I_{1}$. Hence a regular neighborhood of $\gamma$ in $\partial X$ is given by $\gamma \times I_{1} \times I_{2}$ where $I_{2}$ is a small neighborhood of the $\{p t\}$ in $S^{1}=I /(1 \sim 0)$. This neighborhood of $\gamma$ is called the product neighborhood $[\mathbf{K}]$.

Now let us push off $\gamma$ to the boundary of $\partial X-\stackrel{\circ}{\nu}(\gamma)$. Denote the push off of $\gamma$ as $l^{\prime}$. Moreover if we identify $I_{1} \times I_{2}$ as $D^{2}$ and denote $\partial D^{2}$ as $m^{\prime}$, then $\left\{l^{\prime}, m^{\prime}\right\}$ will be a longitude-meridian pair for $\partial(\partial X-\stackrel{\circ}{\nu}(\gamma))$. Then clearly

$$
i_{*}\left[l^{\prime}\right]=[\gamma] \in H_{1}(\partial X-\stackrel{\circ}{\nu}(\gamma) ; \mathbb{R}) .
$$

On the other hand, to find the image of $m^{\prime}$ we observe the following:

Assume that $e \cdot[\gamma]=1$ for some $e \in\left\{a_{1}, b_{1}, a_{2}, b_{2}, \ldots, a_{g}, b_{g}\right\}$. Then we locally have the picture in Figure 3 in a neighborhood of the point where $e$ and $\gamma$ meet.

This proves that

$$
i_{*}\left[m^{\prime}\right]=e-\phi_{*}(e) \in H_{1}(\partial X-\stackrel{\circ}{\nu}(\gamma) ; \mathbb{R}) .
$$

Note that here we can deform $e-m^{\prime}$ into $\phi_{*}(e)$ since the part of $e$ which is not along $m^{\prime}$ lies outside of $\stackrel{\circ}{\nu}(\gamma)$.

Now assume that $e \cdot[\gamma]=-1$ for some $e \in\left\{a_{1}, b_{1}, a_{2}, b_{2}, \ldots, a_{g}, b_{g}\right\}$. Then we locally have a similar picture in a neighborhood of the point where $e$ and $\gamma$ meet, except for the orientations.

This proves that

$$
i_{*}\left[m^{\prime}\right]=\phi_{*}(e)-e \in H_{1}(\partial X-\stackrel{\circ}{\nu}(\gamma) ; \mathbb{R}) .
$$




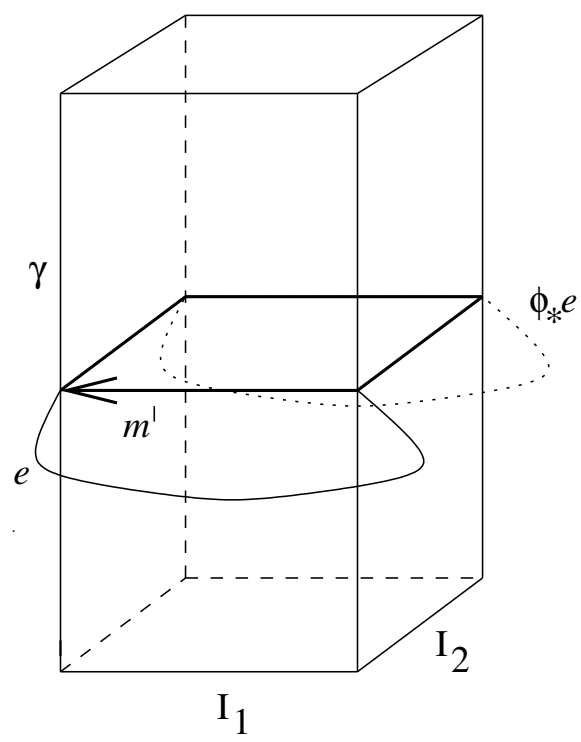

Figure 3.

Since these are local results it follows combining these two observations that

$$
i_{*}\left[m^{\prime}\right]=\frac{e-\phi_{*}(e)}{e \cdot[\gamma]} \in H_{1}(\partial X-\stackrel{\circ}{\nu}(\gamma) ; \mathbb{R}) .
$$

To prove the second part of the theorem we note that if $\gamma$ is a separating curve in $\Sigma_{g}$ then it is homologically trivial. Thus $i_{*}\left[l^{\prime}\right]=0$. This implies that $\operatorname{Ker}\left(i_{*}\right)=\left\langle\left[l^{\prime}\right]\right\rangle$.

Note that, in terms of the bases $\{[l],[m]\}$ of $H_{1}\left(\partial\left(S^{1} \times D^{2}\right) ; \mathbb{R}\right)=H_{1}\left(S^{1} \times\right.$ $\left.S^{1} ; \mathbb{R}\right)$ and $\left\{\left[l^{\prime}\right],\left[m^{\prime}\right]\right\}$ of $H_{1}(\partial(\partial X-\stackrel{\circ}{\nu}(\gamma)) ; \mathbb{R})$, attaching a 2 -handle by -1 framing means that we identify $[l]$ with $\left[l^{\prime}\right]-\left[m^{\prime}\right]$ and $[m]$ with $\left[m^{\prime}\right]$.

So if we transform the $\operatorname{Ker}\left(i_{*}\right)$ to the $\{[l],[m]\}$ plane we see that $\operatorname{Ker}\left(i_{*}\right)=$ $C=W=\langle[l]+[m]\rangle$ which implies that $\sigma\left(X^{\prime}\right)=\sigma(X)-(+1)$ (cf. Theorem 3).

Proposition 5. We use the same notation as in Theorem 4.

(1) Let $\gamma=a_{i}$ for some $i, 1 \leq i \leq g$. Then $H_{1}\left(\partial X-\stackrel{\circ}{\nu}\left(a_{i}\right) ; \mathbb{R}\right)=$ $\left\langle a_{1}, b_{1}, a_{2}, b_{2}, \ldots, a_{g}, b_{g}, b_{i}^{\prime}, t\right| a_{j}=\phi_{*} a_{j}$ for all $j, b_{j}=\phi_{*} b_{j}$ for all $\left.j \neq i, b_{i}^{\prime}=\phi_{*} b_{i}\right\rangle$.

Moreover $i_{*}\left[l^{\prime}\right]=a_{i}$ and $i_{*}\left[m^{\prime}\right]=b_{i}-b_{i}^{\prime}$.

(2) Let $\gamma=b_{i}$ for some $i, 1 \leq i \leq g$. Then $H_{1}\left(\partial X-\stackrel{\circ}{\nu}\left(b_{i}\right) ; \mathbb{R}\right)=$ $\left\langle a_{1}, b_{1}, a_{2}, b_{2}, \ldots, a_{g}, b_{g}, a_{i}^{\prime}, t\right| b_{j}=\phi_{*} b_{j}$ for all $j, a_{j}=\phi_{*} a_{j}$ for all $\left.j \neq i, a_{i}^{\prime}=\phi_{*} a_{i}\right\rangle$.

Moreover $i_{*}\left[l^{\prime}\right]=b_{i}$ and $i_{*}\left[m^{\prime}\right]=a_{i}^{\prime}-a_{i}$. 
Proof. Assume that $\gamma=a_{i}$ for some i, $1 \leq i \leq g$. We first use Van-Kampen's theorem to compute $\pi_{1}\left(\partial X-\stackrel{\circ}{\nu}\left(a_{i}\right)\right)$. Write $\partial X-\stackrel{\circ}{\nu}\left(a_{i}\right)=E_{1} \cup E_{2}$ as follows: Let $E_{1}=\Sigma_{g} \times[0,1 / 2]$ and $E_{2}=\Sigma_{g} \times[1 / 2,1]$. Then glue $\Sigma_{g} \times\{1 / 2\} \subset E_{1}$ with $\Sigma_{g} \times\{1 / 2\} \subset E_{2}$ by the identity map except a neighborhood of $a_{i}$, namely $a_{i} \times I \subset \Sigma_{g}$. Denote the result as $E^{\prime}$. By a trivial calculation we get the following presentation:

$$
\pi_{1}\left(E^{\prime}\right)=\left\langle a_{1}, b_{1}, a_{2}, b_{2}, \ldots, a_{g}, b_{g}, b_{i}^{\prime} \mid \prod_{j=1}^{g}\left[a_{j}, b_{j}\right],\left[a_{i}, b_{i}^{\prime}\right] \prod_{j \neq i}\left[a_{j}, b_{j}\right]\right\rangle .
$$

Finally we Abelianize this presentation after gluing $\Sigma_{g} \times\{0\} \subset E_{1}$ with $\Sigma_{g} \times\{1\} \subset E_{2}$ using the map $\phi$ to get $\partial X-\stackrel{\circ}{\nu}\left(a_{i}\right)$.

$i_{*}\left[l^{\prime}\right]=a_{i}$ and $i_{*}\left[m^{\prime}\right]=b_{i}-b_{i}^{\prime}$ follows from Theorem 4 because $a_{i}$ intersects $b_{j}$ only once iff $i=j$.

Second part is obtained similarly.

\section{The algorithm and examples.}

Suppose that we are attaching a 2-handle along a simple closed curve $\gamma$ to a 4-manifold $M$ with boundary $\partial M \cong \Sigma_{g} \times I /(x, 1) \sim(\phi(x), 0)$, where $\phi$ is a self-diffeomorphism of $\Sigma_{g}$. To compute the signature contribution of this handle we first compute $C=\operatorname{ker} i_{*}$ (cf. Wall's formula) using Theorem 4 and Proposition 5 and then apply Theorem 3. The signature contribution of a 2-handle will depend on the action of $\phi$ on $H_{1}\left(\Sigma_{g}\right)$ and the homology class $[\gamma] \in H_{1}\left(\Sigma_{g}\right)$.

\subsection{Genus 1.}

To illustrate how one can develop an algorithm using our main theorems to calculate the signatures of smooth Lefschetz fibrations, we will give the details of our computation to obtain the well-known result $\sigma(E(1))=-8$ for the elliptic surface $E(1)$.

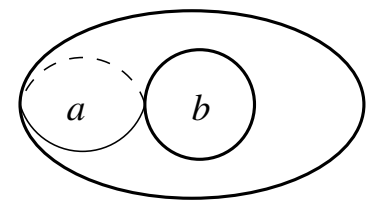

Figure 4.

The global monodromy of $E(1)$ is given by the sequence $(\alpha, \beta)^{6}$ of 12 Dehn twists where $\alpha=D(a)$ and $\beta=D(b)$ denote the positive Dehn twists about the curves $a$ and $b$, respectively (Figure 4).

To build up $E(1)$, we start with a copy of $T^{2} \times D^{2}$ and glue 2-handles along the vanishing cycles $a$ and $b$ in an alternating fashion. (We will use 
the letters $a$ and $b$ also to denote the homology classes of the curves $a$ and $b$, respectively.)

Let $\phi$ denote the monodromy of the boundary of the 4-manifold before we attach a 2-handle.

We take $A, B$ and $C$ as in the proof of Theorem 3 and we apply Proposition 5 to compute $i_{*}\left[l^{\prime}\right]$ and $i_{*}\left[m^{\prime}\right]$. Note that we identify $[l]$ with $\left[l^{\prime}\right]-\left[m^{\prime}\right]$ and $[m]$ with $\left[m^{\prime}\right]$ as in the proof of Theorem 4 .

$\phi=i d e n t i t y$, attach the first handle along $a$,

$i_{*}\left[l^{\prime}\right]=a$ and $i_{*}\left[m^{\prime}\right]=b-b^{\prime}=b-\phi_{*} b=b-b=0$,

$C=\left\langle\left[m^{\prime}\right]\right\rangle=\langle[m]\rangle=B$ and therefore $\sigma(i d, a)=0$.

$\phi=\alpha$, attach the second handle along $b$,

$i_{*}\left[l^{\prime}\right]=b$ and $i_{*}\left[m^{\prime}\right]=a^{\prime}-a=\phi_{*} a-a=0$,

$C=\left\langle\left[m^{\prime}\right]\right\rangle=\langle[m]\rangle=B$ and therefore $\sigma(\alpha, b)=0$.

$\phi=\beta \alpha$, attach the third handle along $a$,

$i_{*}\left[l^{\prime}\right]=a$ and $i_{*}\left[m^{\prime}\right]=b-b^{\prime}=b-\phi_{*} b=b-a=-a$ since $a=\phi_{*} a=a-b$, $C=\left\langle\left[m^{\prime}\right]+\left[l^{\prime}\right]\right\rangle=\langle 2[m]+[l]\rangle$ and therefore $\sigma(\beta \alpha, a)=-1$.

$\phi=\alpha \beta \alpha$, attach the fourth handle along $b$,

$i_{*}\left[l^{\prime}\right]=b$ and $i_{*}\left[m^{\prime}\right]=a^{\prime}-a=\phi_{*} a-a=-b-a=-2 b$ since $b=\phi_{*} b=a$, $C=\left\langle\left[m^{\prime}\right]+2\left[l^{\prime}\right]\right\rangle=\langle 3[m]+2[l]\rangle$ and therefore $\sigma(\alpha \beta \alpha, b)=-1$.

$\phi=\beta \alpha \beta \alpha$, attach the fifth handle along $a$,

$i_{*}\left[l^{\prime}\right]=a$ and $i_{*}\left[m^{\prime}\right]=b-b^{\prime}=b-\phi_{*} b=b-(a-b)=2 b-a=3 b$ since

$a=\phi_{*} a=-b$,

$C=\left\langle\left[m^{\prime}\right]+3\left[l^{\prime}\right]\right\rangle=\langle 4[m]+3[l]\rangle$ and therefore $\sigma(\beta \alpha \beta \alpha, a)=-1$.

$\phi=\alpha \beta \alpha \beta \alpha$, attach the sixth handle along $b$,

$i_{*}\left[l^{\prime}\right]=b=0$ and $i_{*}\left[m^{\prime}\right]=a^{\prime}-a=\phi_{*} a-a=-a-b-a=-2 a-b=-2 a$ since $b=\phi_{*} b=-b$,

$C=\left\langle\left[l^{\prime}\right]\right\rangle=\langle[m]+[l]\rangle$ and therefore $\sigma(\alpha \beta \alpha \beta \alpha, b)=-1$.

$\phi=\beta \alpha \beta \alpha \beta \alpha$, attach the seventh handle along $a$,

$i_{*}\left[l^{\prime}\right]=a=0$ since $a=\phi_{*} a=-a$ and $i_{*}\left[m^{\prime}\right]=b-b^{\prime}=b-\phi_{*} b=$

$b-(-b)=2 b$,

$C=\left\langle\left[l^{\prime}\right]\right\rangle=\langle[m]+[l]\rangle$ and therefore $\sigma(\beta \alpha \beta \alpha \beta \alpha, a)=-1$.

$\phi=\alpha \beta \alpha \beta \alpha \beta \alpha$, attach the eighth handle along $b$,

$i_{*}\left[l^{\prime}\right]=b$ and $i_{*}\left[m^{\prime}\right]=a^{\prime}-a=\phi_{*} a-a=-a-a=-2 a=4 b$ since

$b=\phi_{*} b=-a-b$,

$C=\left\langle-\left[m^{\prime}\right]+4\left[l^{\prime}\right]\right\rangle=\langle 3[m]+4[l]\rangle$ and therefore $\sigma(\alpha \beta \alpha \beta \alpha \beta \alpha, b)=-1$.

$\phi=\beta \alpha \beta \alpha \beta \alpha \beta \alpha$, attach the ninth handle along $a$,

$i_{*}\left[l^{\prime}\right]=a$ and $i_{*}\left[m^{\prime}\right]=b-b^{\prime}=b-\phi_{*} b=b+a=2 a+a=3 a$ since

$a=\phi_{*} a=-a+b$,

$C=\left\langle-\left[m^{\prime}\right]+3\left[l^{\prime}\right]\right\rangle=\langle 2[m]+3[l]\rangle$ and therefore $\sigma(\beta \alpha \beta \alpha \beta \alpha \beta \alpha, a)=-1$. 
$\phi=\alpha \beta \alpha \beta \alpha \beta \alpha \beta \alpha$, attach the tenth handle along $b$,

$i_{*}\left[l^{\prime}\right]=b$ and $i_{*}\left[m^{\prime}\right]=a^{\prime}-a=\phi_{*} a-a=b-a=2 b$ since $b=\phi_{*} b=-a$, $C=\left\langle-\left[m^{\prime}\right]+2\left[l^{\prime}\right]\right\rangle=\langle[m]+[l]\rangle$ and therefore $\sigma(\alpha \beta \alpha \beta \alpha \beta \alpha \beta \alpha, b)=-1$.

$\phi=\beta \alpha \beta \alpha \beta \alpha \beta \alpha \beta \alpha$, attach the eleventh handle along $a$,

$i_{*}\left[l^{\prime}\right]=a$ and $i_{*}\left[m^{\prime}\right]=b-b^{\prime}=b-\phi_{*} b=b-(-a+b)=a$ since $a=\phi_{*} a=b$, $C=\left\langle-\left[m^{\prime}\right]+\left[l^{\prime}\right]\right\rangle=\langle[l]\rangle=A$ and therefore $\sigma(\beta \alpha \beta \alpha \beta \alpha \beta \alpha \beta \alpha, a)=0$.

$\phi=\alpha \beta \alpha \beta \alpha \beta \alpha \beta \alpha \beta \alpha$, attach the twelfth handle along $b$,

$i_{*}\left[l^{\prime}\right]=b$ and $i_{*}\left[m^{\prime}\right]=a^{\prime}-a=\phi_{*} a-a=(a+b)-a=b$,

$C=\left\langle-\left[m^{\prime}\right]+\left[l^{\prime}\right]\right\rangle=\langle[l]\rangle=A$ and therefore $\sigma(\alpha \beta \alpha \beta \alpha \beta \alpha \beta \alpha \beta \alpha, b)=0$.

Therefore by Theorem 3

$$
\begin{gathered}
\sigma(E(1))=\sigma(i d, a)+\sigma(\alpha, b)+\sigma(\beta \alpha, a)+\cdots+\sigma(\alpha \beta \alpha \beta \alpha \beta \alpha \beta \alpha \beta \alpha, b) \\
=0+0-(\underbrace{1+\cdots+1}_{8})+0+0=-8 .
\end{gathered}
$$

\subsection{Genus 2.}

We developed a Mathematica program to compute the signature of a 4-manifold which admits a genus two Lefschetz fibration over $D^{2}$ or $S^{2}$ whose global monodromy is given by any finite sequence of positive Dehn twists $D\left(c_{1}\right), D\left(c_{2}\right), \ldots, D\left(c_{5}\right)$, where $c_{1}, \ldots, c_{5}$ are the curves indicated in Figure 5

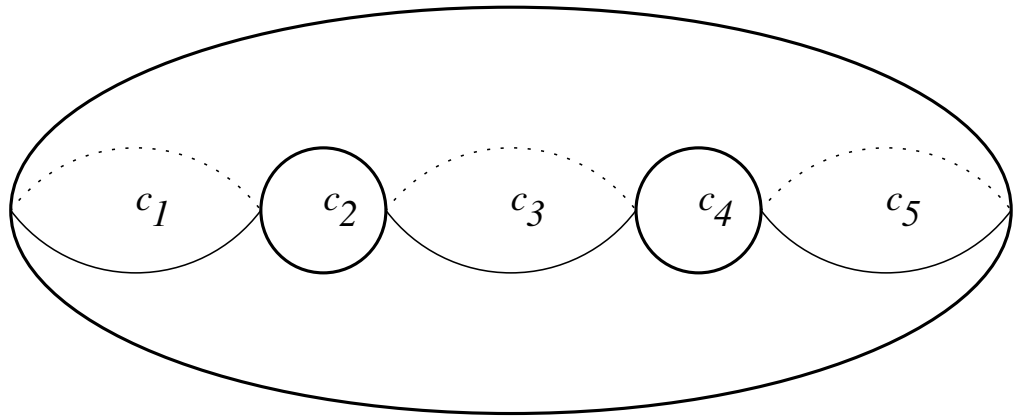

Figure 5.

Let $\zeta_{i}$ denote $D\left(c_{i}\right), 1 \leq i \leq 5$.

It was shown in $[\mathbf{M} 2]$ that $\mathbb{C} P^{2} \# 13 \overline{\mathbb{C} P^{2}}$ admits a smooth Lefschetz fibration of genus two with global monodromy $\left(\zeta_{1}, \zeta_{2}, \zeta_{3}, \zeta_{4}, \zeta_{5}, \zeta_{5}, \zeta_{4}, \zeta_{3}, \zeta_{2}, \zeta_{1}\right)^{2}$. We computed the signature of the total space as

$$
\begin{aligned}
& \sigma\left(\left(\zeta_{1}, \zeta_{2}, \zeta_{3}, \zeta_{4}, \zeta_{5}, \zeta_{5}, \zeta_{4}, \zeta_{3}, \zeta_{2}, \zeta_{1}\right)^{2}\right) \\
& =0+0+0+0-(\underbrace{1+\cdots+1}_{12})+0+0+0+0 \\
& =-12 .
\end{aligned}
$$


One can also compute the signature of the total space starting from a cyclic permutation of the word above as follows.

$$
\begin{aligned}
& \sigma\left(\zeta_{2}, \zeta_{3}, \zeta_{4}, \zeta_{5}, \zeta_{5}, \zeta_{4}, \zeta_{3}, \zeta_{2}, \zeta_{1}, \zeta_{1}, \zeta_{2}, \zeta_{3}, \zeta_{4}, \zeta_{5}, \zeta_{5}, \zeta_{4}, \zeta_{3}, \zeta_{2}, \zeta_{1}, \zeta_{1}\right)= \\
& 0+0+0+0-(\underbrace{1+\cdots+1}_{11})+0+0+0-1+0=-12 .
\end{aligned}
$$

Similarly a genus two Lefschetz fibration on $K 3 \# 2 \overline{\mathbb{C} P^{2}}$ is given in [M2] with the global monodromy $\left(\zeta_{1}, \zeta_{2}, \zeta_{3}, \zeta_{4}, \zeta_{5}\right)^{6}$.

We computed that $\sigma\left(\left(\zeta_{1}, \zeta_{2}, \zeta_{3}, \zeta_{4}, \zeta_{5}\right)^{6}\right)=$

$$
0+0+0+0-(\underbrace{1+\cdots+1}_{9})+0+0+0+0-(\underbrace{1+\cdots+1}_{9})+0+0+0+0=-18 .
$$

Matsumoto [M2] also shows that $\left(\zeta_{1}, \zeta_{2}, \zeta_{3}, \zeta_{4}\right)^{5}$ is the hyperelliptic involution in $\mathcal{M}_{2}$, inducing the relation $\left(\zeta_{1}, \zeta_{2}, \zeta_{3}, \zeta_{4}\right)^{10}=1$.

We computed that $\sigma\left(\left(\zeta_{1}, \zeta_{2}, \zeta_{3}, \zeta_{4}\right)^{10}\right)=0+0+0+0-(\underbrace{1+\cdots+1}_{8})+$

$0+0+1-(\underbrace{1+\cdots+1}_{10})+1+0+0-(\underbrace{1+\cdots+1}_{8})+0+0+0+0=-24$.

Note that the 15th and the 26th 2-handle both contribute +1 to the signature. It is known that the total space of this fibration is homeomorphic but not diffeomorphic to $5 \mathbb{C} P^{2} \# 29 \overline{\mathbb{C} P^{2}}$ (cf. [M2], [F1]).

As a final example we give the signature contributions of the singular fibers in the genus two Lefschetz fibration of $S^{2} \times T^{2} \# 4 \overline{\mathbb{C} P^{2}}$ given in [M2].

$$
\sigma\left(S^{2} \times T^{2} \# 4 \overline{\mathbb{C} P^{2}}\right)=0-1-0-1-1-1+0+0=-4 .
$$

Remark. One can indeed check these numbers using Matsumoto's local signature formula or using the fact that $\sigma\left(X \# \overline{\mathbb{C} P^{2}}\right)=\sigma(X)-1$, for a 4-manifold $X$.

\subsection{Genus 3.}

Let $\mathbb{F}_{2}$ denote the Hirzebruch surface, the holomorphic $\mathbb{C} P^{1}$ bundle over $\mathbb{C} P^{1}$ with a holomorphic section $s_{1}$ of self intersection $-2 . \mathbb{F}_{2}$ also admits a disjoint holomorphic section $s_{2}$ of self intersection 2 . Let $X$ be the two-fold cover of $\mathbb{F}_{2}$, branced over the disjoint union of a smooth curve in $\left|7 s_{1}\right|$ and $s_{2}$. Then $X$ admits a holomorphic Lefschetz fibration $X \rightarrow \mathbb{C} P^{1}$ of genus three obtained by composing the branched cover map with the bundle map $\mathbb{F}_{2} \rightarrow \mathbb{C} P^{1}$. In $[\mathbf{F 2}]$, Fuller gives the global monodromy of this fibration as

$$
\left(\eta_{1}, \eta_{2}, \eta_{3}, \eta_{4}, \eta_{5}, \eta_{6}\right)^{14}
$$

Here $\eta_{1}, \eta_{2}, \ldots, \eta_{9}$ denote the positive Dehn twists about the curves $d_{1}, d_{2}, \ldots, d_{9}$ indicated as in Figure 6. 


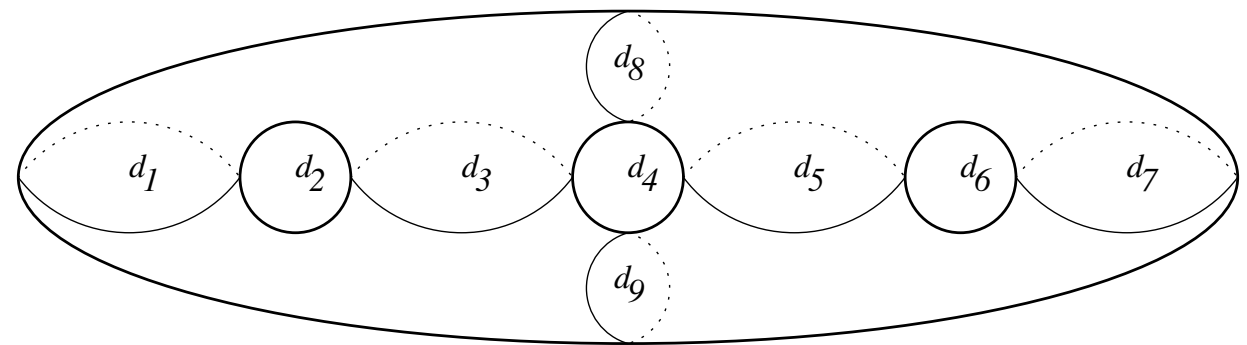

Figure 6.

We computed the signature of this genus three Lefschetz fibrations over $S^{2}$, using our Mathematica program. (The program is available upon request.)

$$
\begin{aligned}
& \sigma\left(\left(\eta_{1}, \eta_{2}, \eta_{3}, \eta_{4}, \eta_{5}, \eta_{6}\right)^{14}\right)= \\
& \underbrace{0+\cdots+0}_{6}-(\underbrace{1+\cdots+1}_{8})+\underbrace{0+\cdots+0}_{4}-(\underbrace{1+\cdots+1}_{8})+\underbrace{0+\cdots+0}_{4} \\
& -1-1+0+0+1-(\underbrace{1+\cdots+1}_{14})+1+0+0-1-1+ \\
& \underbrace{0+\cdots+0}_{4}-(\underbrace{1+\cdots+1}_{8})+\underbrace{0+\cdots+0}_{4}-(\underbrace{1+\cdots+1}_{8})+\underbrace{0+\cdots+0}_{6} \\
& =-48 .
\end{aligned}
$$

Fuller (cf. [F3]) also derives the following word in $\mathcal{M}_{3}$.

$$
\left(\eta_{8}, \eta_{9}, \eta_{4}, \eta_{3}, \eta_{2}, \eta_{1}, \eta_{5}, \eta_{4}, \eta_{3}, \eta_{2}, \eta_{6}, \eta_{5}, \eta_{4}, \eta_{3},\left(\eta_{1}, \eta_{2}, \eta_{3}, \eta_{4}, \eta_{5}, \eta_{6}\right)^{10}\right)
$$

We computed that

$$
\begin{aligned}
& \sigma\left(\left(\eta_{8}, \eta_{9}, \eta_{4}, \eta_{3}, \eta_{2}, \eta_{1}, \eta_{5}, \eta_{4}, \eta_{3}, \eta_{2}, \eta_{6}, \eta_{5}, \eta_{4}, \eta_{3},\left(\eta_{1}, \eta_{2}, \eta_{3}, \eta_{4}, \eta_{5}, \eta_{6}\right)^{10}\right)\right) \\
& =-42 .
\end{aligned}
$$

The four manifold with the global monodromy given as above is not very familiar and it is our only example where we can not use any other method than ours to calculate the signature. The Lefschetz fibration is not hyperelliptic, for example, otherwise the local signature formula [E] would yield $\sigma=74(-4 / 7)$ which is not an integer! It is not known whether this fibration is holomorphic or not.

Corollary 6. There exist two genus three Lefschetz fibrations with the same Euler characteristic but having different signatures.

Proof. Let $M_{1}$ and $M_{2}$ be the 4-manifolds with global monodromies

$$
\left(\eta_{1}, \eta_{2}, \eta_{3}, \eta_{4}, \eta_{5}, \eta_{6}\right)^{14}
$$


and

$\left(\eta_{8}, \eta_{9}, \eta_{4}, \eta_{3}, \eta_{2}, \eta_{1}, \eta_{5}, \eta_{4}, \eta_{3}, \eta_{2}, \eta_{6}, \eta_{5}, \eta_{4}, \eta_{3},\left(\eta_{1}, \eta_{2}, \eta_{3}, \eta_{4}, \eta_{5}, \eta_{6}\right)^{10}\right)$ respectively.

Let $\#_{n} M$ denote the $n$-fold fiber sum of $M$ by itself. Then $e\left(\#_{37} M_{1}\right)=$ $e\left(\#_{42} M_{2}\right)$ but $\sigma\left(\#_{37} M_{1}\right)=-1776$ and $\sigma\left(\#_{42} M_{2}\right)=-1764$.

Remark. Following the language in $[\mathbf{S m}]$, we say that two Lefschetz fibrations are combinatorially equivalent if they have the same fiber genus and the same number of each conjugacy type of singular fibers. The corollary above shows that signature is not an invariant of the combinatorial equivalence class of a Lefschetz fibration. Moreover there is not necessarily a hyperelliptic Lefschetz fibration in each combinatorial equivalence class.

\section{Some applications.}

First we give an immediate application of Theorems 3 and 4 .

Corollary 7. Let $M$ be a 4-manifold which admits a genus $g$ Lefschetz fibration over $D^{2}$ or $S^{2}$. Let $n$ and $s$ be the numbers of nonseparating and separating vanishing cycles in the global monodromy of this fibration, respectively. Then $\sigma(M) \leq n-s$.

Proof. Suppose that we build up the 4-manifold $M$ from $\Sigma_{g} \times D^{2}$ by attaching 2-handles. By Theorem 4, every time we attach a 2-handle along a separating curve the signature of the resulting 4-manifold will be one less than the signature of the 4-manifold before we attach the 2-handle. Thus Theorem 3 implies the upper bound $n-s$ on the signature.

Remark. Define $c_{1}^{2}(M)=3 \sigma(M)+2 \chi(M)$ and $\chi_{h}(M)=\frac{1}{4}(\sigma(M)+\chi(M))$ for a closed symplectic 4-manifold $M$. Note that $\sigma(M) \leq n+s=\chi(M)+$ $4 g-4$ trivially implies $c_{1}^{2} \leq 10 \chi_{h}+2 g-2$ for a genus $g$ Lefschetz fibration over $S^{2}$.

Corollary 8. There is no hyperelliptic Lefschetz fibration (of any genus) over $S^{2}$ with only reducible singular fibers. (Here reducible means that the local monodromy corresponding to the singular fiber is a Dehn twist about a separating curve.)

Remark. In particular, this proves that a product of positive Dehn twists about separating curves can not be equal to the identity in $\mathcal{M}_{2}$, which is a result of Mess [Me].

Proof. Let $M$ be a 4-manifold which admits a Lefschetz fibration of genus $g$ over $S^{2}$ with global monodromy $\left(D\left(\gamma_{1}\right), \ldots, D\left(\gamma_{s}\right)\right)$, where $s=\sum_{h=1}^{\left[\frac{g}{2}\right]} s_{h}$ and $\gamma_{i}$ is separating for each $\mathrm{i}, 1 \leq i \leq s$. Then, by the local signature 
formula,

$$
\sigma(M)= \begin{cases}\sum_{h=1}^{\left[\frac{g}{2}\right]}\left(\frac{4 h(g-h)}{2 g+1}-1\right) s_{h} \geq 0 & \text { if } g \geq 3 \\ -s / 5 & \text { if } g=2 .\end{cases}
$$

But on the other hand $\sigma(M)=-s$ according to Theorem 4. Hence $s=0$. (This is trivially true for $g=1$ since any vanishing cycle on a torus is nonseparating.) This proves the desired result since we assume (by definition) that there exists at least one singular fiber in each Lefschetz fibration.

Next we combine our results with the local signature formula for the hyperelliptic Lefschetz fibrations to give an upper bound for the signatures of these fibrations.

Corollary 9. Let $M$ be a 4-manifold which admits a hyperelliptic Lefschetz fibration of genus $g$ over $S^{2}$. Let $n$ and $s$ be the numbers of nonseparating and separating vanishing cycles in the global monodromy of this fibration, respectively. Then $\sigma(M) \leq n-s-4$.

Remark. This inequality is not necessarily sharp.

Proof. We first note that we can improve the inequality

$$
\sigma(M) \leq n-s
$$

given in Corollary 7 to

$$
\sigma(M) \leq n-s-1
$$

for hyperelliptic Lefschetz fibrations as follows:

Suppose that we attach the first 2-handle along a nonseparating curve. We can always assume this because $n \geq 1$ (since we proved in Corollary 8 that $n \neq 0$ ) and we can cyclically permute the vanishing cycles in the global monodromy of a Lefschetz fibration. Moreover we can easily show that if we start attaching handles along a nonseparating curve then the signature of the resulting 4-manifold (after attaching the very first handle) will be the same as $\sigma\left(\Sigma_{2} \times D^{2}\right)$, which is zero.

Next note that $\sigma(M) \leq n-s-1$ is equivalent to

$$
4 \sum_{h=1}^{\left[\frac{g}{2}\right]} h(g-h) s_{h} \leq(3 g+2) n-(2 g+1)
$$

using the local signature formula.

Assume that $g$ is odd. Endo $[\mathbf{E}]$ proves that

$$
n+4 \sum_{h=1}^{\left[\frac{g}{2}\right]} h(2 h+1) s_{h} \equiv 0(\bmod 4(2 g+1)) .
$$


Hence

$$
n=4 c(2 g+1)-4 \sum_{h=1}^{\left[\frac{g}{2}\right]} h(2 h+1) s_{h}
$$

for some integer c. Substituting into the inequality above (and dividing by 4) we get

$$
\sum_{h=1}^{\left[\frac{g}{2}\right]} h(g-h) s_{h} \leq(3 g+2)\left[c(2 g+1)-\sum_{h=1}^{\left[\frac{g}{2}\right]} h(2 h+1) s_{h}\right]-\frac{1}{4}(2 g+1) .
$$

Hence

$$
\sum_{h=1}^{\left[\frac{g}{2}\right]} h(g-h) s_{h} \leq(3 g+2)\left[c(2 g+1)-\sum_{h=1}^{\left[\frac{g}{2}\right]} h(2 h+1) s_{h}\right]-\frac{1}{4}(2 g+2)
$$

since $2 g+1 \equiv 3(\bmod 4)$.

But this inequality, in turn, implies that

$$
4 \sum_{h=1}^{\left[\frac{g}{2}\right]} h(g-h) s_{h} \leq(3 g+2) n-(2 g+2)
$$

which is equivalent to

$$
\sigma(M) \leq n-s-1-\frac{1}{2 g+1} .
$$

Since $\sigma(M)$ is an integer,

$$
\sigma(M) \leq n-s-2 .
$$

Iterating the same argument, we obtain

$$
\sigma(M) \leq n-s-4 .
$$

(We use $2(2 g+1) \equiv 2(\bmod 4)$ and $3(2 g+1) \equiv 1(\bmod 4)$.)

Similarly, if $g$ is even, then one can use the corresponding result by Endo:

$$
n+4 \sum_{h=1}^{\left[\frac{g}{2}\right]} h(2 h+1) s_{h} \equiv 0(\bmod 2(2 g+1)) .
$$

(Note that $2(3 g+2)(2 g+1) \equiv 0(\bmod 4)$, if $\mathrm{g}$ is even.)

The following is a result concerning the geography of the hyperelliptic Lefschetz fibrations, which follows easily from Corollary 9.

Corollary 10. (1) The total space of a genus two Lefschetz fibration over $S^{2}$ satisfies

$$
c_{1}^{2} \leq 6 \chi_{h}-3 .
$$


(2) The number of singular fibers in a genus two Lefschetz fibration over $S^{2}$ can not be equal to 5,6,11 or 12 and in particular, the minimal number of singular fibers in a genus two Lefschetz fibration over $S^{2}$ is 7 or 8.

(3) The total space of a genus three hyperelliptic Lefschetz fibration over $S^{2}$ satisfies

$$
c_{1}^{2} \leq 7.25 \chi_{h}-2.75 \text {. }
$$

Remarks. (1) In particular, the signature of a smooth 4-manifold which admits a hyperelliptic Lefschetz fibration of genus $g \leq 3$ over $S^{2}$ is negative.

(2) Similar inequalities can be obtained for genus $g \geq 4$ hyperelliptic Lefschetz fibrations over $S^{2}$.

\section{Final remark.}

Given a product of positive Dehn twists in the mapping class group of a genus $g$ surface, we can construct a symplectic 4-manifold which admits a Lefschetz fibration over $D^{2}$, as we have studied in this paper. A natural generalization is to allow negative Dehn twists also. These fibrations are called achiral Lefschetz fibrations. Our technique clearly extends to compute the signatures of these fibrations.

\section{References}

[BH] J.Birman and H.Hilden, On mapping class groups of closed surfaces as covering spaces, Advances in the Theory of Riemann Surfaces, Annals of Math. Studies, 66, Princeton Univ. Press, (1971), 81-115, Zbl 217.48602.

[D] S. Donaldson, Lefschetz fibrations in symplectic geometry, Doc. Math. J. DMV., Extra Volume, ICMII (1998), 309-314, MR 99i:57044, Zbl 909.53018.

[E] H. Endo, Meyer's signature cocyle and hyperelliptic fibrations, Math. Ann., 316 (2000), 237-257, MR 2001b:57047, Zbl 0948.57013.

[F1] T. Fuller, Diffeomorphism types of genus 2 Lefschetz fibrations, Math. Ann., 311(1) (1998), 163-176, MR 99f:57035, Zbl 905.57014.

[F2] , Hyperelliptic Lefschetz fibrations and branched covering spaces, Pacific J. Math., 196 (2000), 369-393, CMP 1800582.

[F3] , private communication.

[GS] R. Gompf and A. Stipsicz, An introduction to 4-manifolds and Kirby calculus, AMS Graduate Studies in Mathematics, 20 (1999).

[K] A. Kas, On the handlebody decomposition associated to a Lefschetz fibration, Pacific J. Math., 89 (1980), 89-104, MR 82f:57012, Zbl 457.14011.

[L] T-J. Li, Symplectic Parshin-Arakelov inequality, Internat. Math. Res. Notices, 18 (2000), 941-954, CMP 1792283.

[M1] Y. Matsumoto, On 4-manifolds fibered by tori II, Proc. Japan Acad., 59A (1983), 100-103, MR 84j:57010b, Zbl 532.55020. 
[M2] Lefschetz fibrations of genus two - a topological approach, Proceedings of the 37th Taniguchi Symposium on Topology and Teichmüller Spaces, ed. Sadayoshi Kojima et al., World Scientific (1996), 123-148, MR 2000h:14038, Zbl 921.57006.

[Me] G. Mess, The Torelli groups for genus 2 and 3 surfaces, Topology, 31(4) (1992), 775-790, MR 93k:57003, Zbl 772.57025.

[Sm] I. Smith, Lefschetz fibrations and the Hodge bundle, Geom. Topol., 3 (1999), 211233, MR 2000j:57059, Zbl 929.53047.

[St1] A. Stipsicz, A note on the geography of symplectic manifolds, Turkish J. Math., 20 (1996), 135-139, MR 97m:57035, Zbl 876.57039.

[St2] _ Erratum to: Chern numbers of certain Lefschetz fibrations, Proc. Amer. Math. Soc., 128(9) (2000), 2833-2834, CMP 1766607.

[St3] - On the number of vanishing cycles in Lefschetz fibrations, Math. Res. Lett., 6 (1999), 449-456, MR 2000g:57046, Zbl 955.57026.

[W] C.T.C. Wall, Non-additivity of the signature, Inventiones Math., 7 (1969), 269-274, MR 39 \#7615, Zbl 176.21501.

Received January 8, 2000 and revised March 7, 2000.

Department of Mathematics

Michigan State University

EAST LANSING, MI 48824-1028

E-mail address: bozbagci@math.msu.edu 